\title{
Mehrwertanbieter im Massengeschäft
}

Manche Unternehmen in Europa konzentrieren sich auf Mehrwertangebote, sie lösen die Probleme der Kunden und tragen zu ihrem Erfolg bei. Elemente sind umfassende Kundenbetreuung und Services bis zu Spitzentechnologien. Durch günstigere Anbieter, zum Beispiel auch asiatische Billiganbieter, sind diese Unternehmen herausgefordert. Deshalb suchen sie nach geeigneten Strategien. Der Beitrag zeigt die Herausforderungen und strategische Optionen.

Christian Belz 
Das Thema Mehrwertangebote ist nicht neu, trifft aber Märkte und Unternehmen zu verschiedenen Zeiten. Für manche Anbieter sind die Herausforderungen sehr aktuell, besonders in der Schweiz noch akzentuiert durch die große Wechselkursverschiebung am Anfang des Jahres.

Die Fallbeispiele im folgenden Beitrag lassen sich bedingt vergleichen. Wie für jede strategische Initiative sind Markt-, Wettbewerbs- und Kundenanalysen ebenso wichtig wie abzuklären, welche Stärken und Ressourcen ein Unternehmen einbringen kann. Die Thematik beschäftigt aber viele Branchen, die Fälle reichen von der Maschinenindustrie und Dienstleistungen bis zum Einzelhandel. Dieser Beitrag konzentriert sich auf den B2B-Bereich.

\section{Grenzen des Mehrwerts}

Die Positionierung als Premiumanbieter kann aus verschiedenen Gründen problematisch werden. Auch anspruchsvolle Kunden begnügen sich in ihrer Beschaffung mit schlanken Angeboten, zudem kaufen sie einmal mit umfassenderer Unterstützung ein, ein anders Mal wählen sie sehr schlanke und günstige Angebote. Die Qualitätskriterien können dabei immer sehr hoch sein, die „Fitness for use“ wird aber je nach Situation verschieden bewertet.

Die größte Leistungsfähigkeit eines Angebotes bedeutet noch nicht automatisch, dass damit die Anforderungen des Kunden erfüllt sind. Typisch der Kunde eines Maschinenherstellers: „Was nützt mir die höchste Produktivität ihrer Maschine, wenn ich damit meinen Bedarf bereits in zwei Tagen pro Woche produzieren kann?" Errechnete Pay-back-Zeiten für eine Investition spielen bei einer ungenügenden Auslastung der Maschinen kaum eine Rolle. Kurze Umstellungszeiten, hohe Bearbeitungsgeschwindigkeit und Langlebigkeit spielen eine kleinere Rolle. Allenfalls ist dem Kunden aber die Flexibilität für die Produktion verschiedener Werkstücke wichtiger. Der Kunde wählt auch, was gut genug für ihn ist, um selbst im Kostendruck gegenüber seinen Kunden besser zu bestehen und eigene Gewinne zu steigern. So verlagern manche Kunden ihre Einkaufsentscheidungen von Fachabteilungen auf das Einkaufsmanagement oder sie nutzen zunehmend Ausschreibungen, um ihre Einkaufsmacht und den Wettbewerb zwischen möglichen Lieferanten zu steigern.

Manche Premiumanbieter sind mehr und mehr nur noch für spezifische Kunden interessant. Ihr Markt wird enger und der Aufwand zur Individualisierung für Kunden steigt. Gleichzeitig entwickeln sich günstigere Anbieter in den Bereich hochwertiger Leistungen und gewinnen damit auch Kunden und Aufträge im High End des Marktes.

Premium- und Hochleistungsanbieter erhalten von neuen Anbietern mit schlanken Geschäftsmodellen Konkurrenz. Dazu gehören oft auch preisaggressive, fernöstliche Anbieter, die sich über mehrere Jahre auf den Gewinn von Marktanteilen konzentrieren. Teilweise spielen auch kleine und flexible Wettbewerber eine Rolle, die nicht durch hohe Fixkosten und lange Entscheidungswege der Konzerne behindert werden. Typisch ist der Wettbewerb im Bereich der After Sales Services, bei welchem sich kleine und lokale, selbstständige Anbieter für den Service von Maschinen mit hohen
Prof. Dr. Christian Belz ist Ordinarius für Marketing an der Universität St. Gallen und Direktor am Institut für Marketing.
Christian Belz

Universität St. Gallen, St. Gallen, Schweiz

E-Mail: christian.belz@unisg.ch 
Populationen im Markt konzentrieren. Sie werden von den ursprünglichen Maschinenanbietern oft als Piraten bezeichnet (Baumbach 2004). Schließlich führte Prahalad mit seinen Büchern zur Bottom of the Pyramid (2004) auf die umfassende Diskussion, ob sich Angebote für die Millionen von armen Menschen auf der Welt erfolgreich nutzen und damit den Betroffenen helfen lässt. Vunder (2008) konkretisierte den Ansatz für einen Chemiekonzern in Indien. Sie zeigte dabei, dass es gilt, sich in multiplen Märkten zu bewegen und nicht Weltstrategien auszurollen.

\section{Kritische Reaktion von}

\section{bestehenden Unternehmen}

Warum geraten Marktführer ins Abseits? Weshalb reagieren Anbieter im Premiumbereich oft nicht auf neue Geschäftsmodelle und bahnbrechende Innovationen? Christensen et al. (2013) beschreiben in ihrem Innovators Dilemma eindrücklich, dass auch Anbieter im Premiumbereich oft auf bahnbrechende Innovationen nicht reagieren können. Davon zeugen ihre Beispiele zu Marktführern, die schließlich ins Abseits fielen. Diese Innovationen werden meist durch kleinere Newcomer lanciert, ihre Leistungsfähigkeit ist begrenzt und am Anfang nur für kleine Teilsegmente gut genug. Sie kämpfen mit den üblichen Anlaufschwierigkeiten und sie erobern erst später Kunden, die sich bisher mit den angestammten Lösun- gen eindeckten. Die Zusammenhänge zeigt Abbildung 1. Die führenden Unternehmen scheitern dabei nicht, weil sie nicht richtig geführt werden. Im Gegenteil konzentrieren sie sich in ihren Strategien und Maßnahmen auf die ertragreichsten Potenziale und dazu gehören schlechtere und billigere Leistungen oder Randsegmente im Markt definitiv nicht.

Erfolg ist bekanntlich die verbreitete Ursache für Misserfolg. Hochleistungsanbieter entwickeln nicht selten eine Überheblichkeit gegenüber den Wettbewerbern und auch Kunden. Zu gut für den Kunden zu sein, scheint eine zulässige Erklärung zu sein, nur bleibt es bei einem Misserfolg. Hochmut kommt vor dem Fall, um ein weiteres geflügeltes Wort zu erwähnen. Plötner (2012, S. 50) bringt es so auf den Punkt: „Westliche Technologie-Unternehmen begegneten dem Aufstieg asiatischer Wettbewerber bisher überwiegend mit Überheblichkeit und Hilflosigkeit. Ihre Manager haben sich zunächst über das vermeintlich rückständige Qualitätsniveau der Produkte mokiert, später dann wurde das überdurchschnittliche Wachstum der neuen Wettbewerber nahezu resigniert als unvermeidbar hingenommen.“

Der Pionier des Marketings Theodor Levitt (1966, S. 63 ff.) schilderte den Prozess bereits anschaulich. Zuerst werden Wettbewerber mit Innovationen belächelt; dann wird festgestellt, dass sie zwar noch im Markt sind, aber die Marktanteile sind zu vernachlässigen; schließlich wird dann bemerkt,

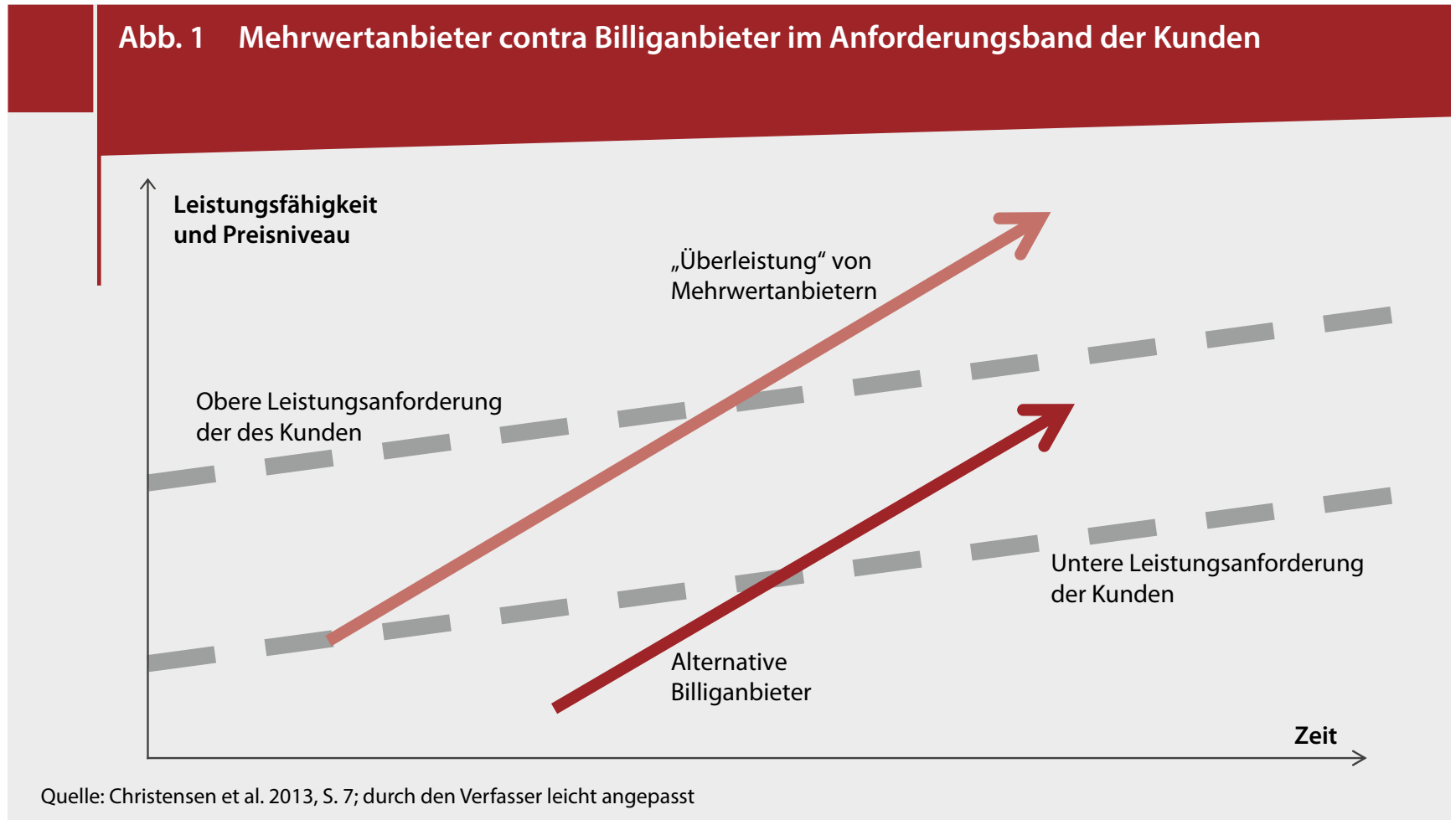


dass sich die neuen Angebote etablieren, aber die Nische scheint jetzt bereits besetzt, irgendwann werden die Verantwortlichen nervös, wenn diese neuen Anbieter weiter wachsen, und es wird ein Crashprogramm im eigenen Unternehmen lanciert, um der Neuerung zu begegnen.

In vielen Märkten ist es üblich, die Strukturen in einer Pyramide von High- bis Low-End-Geschäft darzustellen (siehe Abbildung 2). Es gibt also offenbar ein Oben und Unten im Markt, was häufig eine Wertung einschließt. Häufig wird der untere Bereich auch despektierlich als „Bread and Butter“-Geschäft bezeichnet. Auch erfasst die Pyramide nur eine Dimension, sie entspricht nicht der Vielfalt der Qualitätskriterien für Kunden. Wenn sich Märkte in Luxus und Billig polarisieren, so machten die Anbieter Fehler, weil es ihnen nicht gelang, den attraktiven mittleren Markt auszuloten. Die Mitte ist attraktiv (Belz 2009, S. 77 ff.).

Die knappen Anforderungen von kostenbewussten Kunden $\mathrm{zu}$ erfüllen, ist ebenso schwierig, wie im Luxusbereich aktiv zu sein; wahrscheinlich sogar noch anspruchsvoller. Sich gönnerhaft mit den Billigangeboten zu befassen, mobilisiert eindeutig die erforderliche Kraft nicht. Wohl deshalb suchen Anbieter auch nach attraktiven Bezeichnungen für das Volumengeschäft. Ein Beispiel ist Value Segment (was aber mögliche Verwechslungen mit Mehrwertangeboten einschließt). Teilweise wird SMART als Abkürzung für „simple, maintenance-friendly, affordable, reliable und timely to the market" (Plötner 2012, S. 51) verwendet. Typische Bezeichnungen sind auch Eco, Esenza, Essential, Basic, Focus oder ähnlich. Etwas philosophischer auch De Saint-Exupéry (2000, S. 56): „Vollkommenheit entsteht offensichtlich nicht dann, wenn man nichts mehr hinzuzufügen hat, sondern wenn man nichts mehr wegnehmen kann."

Etablierte Premiumanbieter stoßen also an die Grenzen ihres Wachstums. In den angestammten Märkten mit den bisherigen Leistungen ist das Potenzial begrenzt. Deshalb überdenken mehr und mehr Anbieter ihre Strategie und befassen sich damit, wie sie neben dem bestehenden PremiumGeschäft auch weit günstigere Lösungen anbieten könnten.

\section{Strategische Optionen}

Wie kann sich ein etablierter Mehrwertanbieter im Volumenund Standardgeschäft engagieren? Wir erkennen grundsätzlich vier Möglichkeiten:

1. Getrenntes Unternehmen: Grundsätzlich kann ein Unternehmen im Bereich Spitzentechnologie oder Mehrwert einen weiteren Anbieter aufkaufen, um das Volumengeschäft über ein getrenntes Unternehmen zu bearbeiten. Damit wird die Entscheidung gefällt, die Märkte auf zwei Ebenen anzugehen. Die bestehende Positionierung soll aber nicht verwässert werden. Kritisch ist dabei, ob es gelingt, mit dem Volumenanbieter im Gruppenverbund konsequent und erfolgreich vorzugehen, es also wirksam vom bestehenden Geschäft zu trennen und damit die Konflikte bei Mitarbeitenden und Kunden zu minimieren. Zwar tritt ein neues Unternehmen mit günstigeren Preisen in Konkurrenz zum angestammten Geschäft, eine Kannibalisierung in der eigenen Organisation wird aber vermieden. Beide Unternehmen haben den Freiraum, um sich $\mathrm{zu}$ entwickeln, und es wird nur eine grobe Arbeitsteilung gesucht. Beispielsweise sind digitale Lösungen und E-Business auch im Hochpreissegment möglich und sollten nicht nur für das Massengeschäft erwägt werden. Zudem wirken sich Probleme in einem Geschäft nur über die Gruppe auf den Erfolg aus, damit lassen sich manche weiteren Risiken begrenzen. Eindrücklich ist das Beispiel von Mettler Toledo für diesen Ansatz.

\section{Mettler Toledo und Ohaus als getrennte Anbieter für Mehrwert- und Volumengeschäft}

Mettler Toledo (mit dem Hauptsitz in CH-Greifensee) ist in verschiedenen Teilmärkten weltweit Nummer eins oder zwei. Der Gesamtumsatz für 2014 betrug rund 2,5 Milliarden USDollar. Produkte und Applikationen benennt das Unternehmen mit Wägen im Labor, Analytische Instrumente, Pipetten und Pipettenspitzen, AutoChem, Prozessanalytik, Wägen in der Industrie. Services umfassen maßgeschneiderte Servicepläne für Kunden, Kalibrierservices, Technischer und Software Support, Installation und Inbetriebnahme, Produktinspektion und Solution Consulting. Kundenbranchen reichen von Chemie/Pharma und Biotech, Nahrungsmittel und Getränke, Rohstoffe und Schmuck, Zellstoff/Papier/Textil, Maschinen/Elektro/KfZ, OEM und Systemintegratoren, Transport/Logistik und Wasser/Energie, Einzelhandel bis zum Bildungswesen und der öffentlichen Hand.

Mettler Toledo ist ein professioneller Problemlöser für Kunden. Die Services sind umfassend und die Kunden erhalten mehrheitlich segmentbezogene Dokumentationen und Services, die sich auf ihre spezifischen Herausforderungen und Lösungen konzentrieren. Den Kunden werden kompetente und individuelle Entscheidungshilfen gegeben und sie erkennen rasch, ob sich eine Investition für sie lohnt. So lassen sich für manche Systeme ausgezeichnete Pay-back-Zeiten von einem bis drei Jahren belegen. 
Strategisch setzt sich Mettler Toledo das Ziel, auch im Volumen- und Standardmarkt zu wachsen. Dieser Bereich wurde unter der Marke Ohaus völlig getrennt aufgebaut und geführt (das Unternehmen Ohaus (USA) wurde vor einigen Jahren übernommen). Volumenmarketing, eigene Sortimente mit kostengünstigen Standardprodukten, Low Cost Operation, indirekte Distribution, optimierte und zentrale Logistik und einfache Verkaufsprozesse prägen den Ansatz eines schnellen und schlanken Marketings (mit Internet, Fax, Phone und E-Mail). Synergien gibt es allenfalls noch im Bereich der Forschung und Entwicklung und der Gruppenführung. Die Kunden erkennen zwischen den beiden Firmen kaum Zusammenhänge.

Ein anderes Unternehmen zu kaufen und auf Volumenmärkte auszurichten, ist plausibel. Getrennte Vorgehensweisen lassen sich aber auch mit Kooperationen realisieren und ebenso ist es denkbar, auch ein neues Unternehmen zu gründen und selbst aufzubauen. Allerdings wird der große Zeitbedarf rasch zum kritischen Faktor.

Außerhalb des B2B-Bereichs schaffte es der schweizerische Uhrenkonzern Swatch, in einem großen Portfolio von Unternehmen alle Marktbereiche von der bekannten Swatch bis
Breguet erfolgreich zu bearbeiten. Der Retter der schweizerischen Uhrenindustrie Nicolas Hayek pflegte jeweils aufzuzeigen, dass die Uhrenindustrie im Luxus nur erfolgreich bleiben kann, wenn sie auch das Massengeschäft beherrscht. Interessant ist auch die Accor Hotelgruppe, die in vier Stufen vom Luxusbereich (Sofitel) bis zu Economy (Hotel F1) erfolgreich tätig ist (Plötner 2012, S. 52).

Manchmal wollen Unternehmen mit ihren Volumenangeboten besonders neue Wachstumsmärkte wie Brasilien, Indien oder China erschließen. In gewissen Fällen sind dann diese schlanken Angebote wider Erwarten auch für europäische Märkte oder die USA und weitere Länder besonders attraktiv.

2. Transformation: Die Anforderungen der Kunden und die Bedingungen in den Märkten können sich auch so stark verschieben, dass sich ein erfolgreicher Mehrwertanbieter neu auf ein Volumengeschäft umorientiert. Diese Transformation ist grundsätzlich, anspruchsvoll und risikoreich. Auch hier wird (wie beim getrennten Unternehmen) davon ausgegangen, dass sich die Geschäftsmodelle von Mehrwert und Volumen nicht kombinieren lassen. Die Spielregeln sind zu verschieden. Ein erfolgreiches Beispiel zu dieser Option lieferte eine spezielle Sparte von BASF.

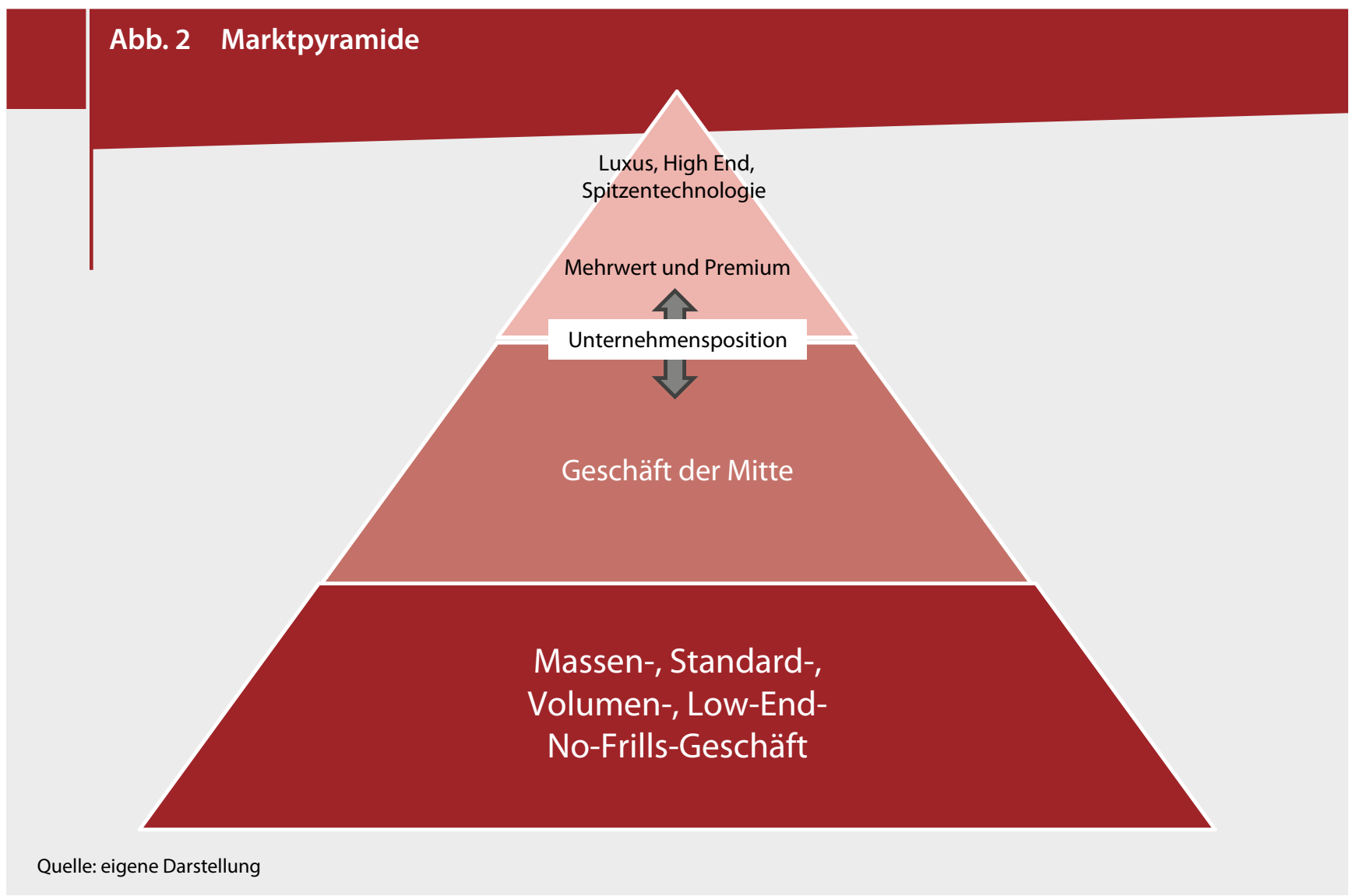


The ABS Story: Terluran ${ }^{\circledR}$ absolutely successful - die Commodity Strategie (Glü ck 2007)

Das Erfolgsbeispiel von BASF betrifft den Bereich der „engineering plastics". Es handelt sich um Copolymere, die Acrylonitrile, Butadien und Styrene enthalten (deshalb ABS). Die Granulate werden beispielsweise von Spritzgießern zu Gehäusen für Waschmaschinen, Staubsauger, Computer, Spielzeug und Sportgeräte, Autoarmaturen und so weiter genutzt. Im Vergleich zu anderen Produkten überzeugt ABS durch Oberflächenqualität, Farben und mechanische Belastbarkeit und Verarbeitbarkeit.

Die Ausgangslage: Nach dem Jahr 2000 verschärfte sich der internationale Wettbewerb besonders durch reagible und günstige asiatische Hersteller. Im globalen ABS-Markt stieg der Anteil der asiatischen Märkte auf 72 Prozent. Vorerst versuchten die meisten europäischen Anbieter und auch BASF, ihre Strategien der vielfältigen Nischenangebote und den Kundensupport (aus den 90er Jahren) zu intensivieren. Zahlreiche vorgefärbte und spezifische ABS-Typen wurden angeboten und der Kunde durch Engineering und Services unterstützt. Die vielfältigen Herausforderungen und Anstrengungen bewirkten zu wenig Erfolg, die Problematik wurde chronisch und durch schlechte Zahlen dieses Bereichs stieg der Druck des Top Managements drastisch. Krisensituationen sind Ausgangslage für eingreifende Veränderungen.

Die Komplexität der Produkte und Dienstleistungen überbordete. Gestützt auf ihr Geschäftsmodell der Spezialitäten führte BASF 2001 fünf ABS-Produktlinien mit mehr als 1.500 Produkten und zahlreichen Verpackungsvarianten.

Die wichtigsten Entscheidungskriterien für den Kunden, wie Produktkonsistenz, zuverlässige Lieferung und Verfügbarkeit sowie kompetitive Preise, wurden durch BASF eher schlecht erfüllt, verursacht durch die eigene Komplexität der Herstellung und der Logistik von Spezialitäten. In verzichtbaren oder weniger wichtigen Bereichen wie Produktentwicklung, Unterstützung der Kundenprozesse, Unterstützung in der Komponentenentwicklung und verständliches Produktportfolio konzentrierten sich die früheren Anstrengungen von BASF. Die kurze Analyse lautete: Mismatch von Value Proposition und realen Kundenbedürfnissen sowie unflexible und teure Strukturen.

Diese Herausforderungen sind typisch für weitere Bereiche von BASF und viele weiteren Unternehmen.

Die neue Strategie: Die neue Strategie stützte sich 2001 auf Analysen erfolgreicher Anbieter im Massengeschäft. Beispiele waren Ryan Air, Dell, Microsoft, Aldi, McDonald's, Formule 1, Ikea oder CHI MEI. Drei Bausteine prägen die neue

\section{Kernthesen}

1. Für manche Premium- und Mehrwert-Anbieter werden die Märkte zu eng und die Billigkonkurrenz wächst.

2. Volumenmärkte erfordern ein hoch professionelles Vorgehen und sind keinesfalls minderwertig.

3. Optionen für Mehrwert-Unternehmen im Volumengeschäft sind 1. getrennte Unternehmen, 2. Transformation, 3. Differenzierung und 4. erweiterte Preisspielräume für gezielte Angebote oder Kundenalternativen.

4. Geschäfte für Mehrwert und Volumen sind anspruchsvoll parallel zu führen. Es gibt mehrere Variablen, um die Geschäfte zu verbinden oder zu trennen.

5. Mit einem Preisspielraum von 40 Prozent gelingt es, verschiedene Qualitätsbedürfnisse von Kunden auf hohem Niveau zu erfüllen und der bisherigen Strategie treu zu bleiben.

Strategie: 1) Wichtigste Kundenbedürfnisse, 2) klar definiertes und implementiertes Commodity-Geschäftsmodell, 3) stringente Kommunikation der Value Proposition für das neue Angebot im Markt.

Das Commodity-Modell konzentriert sich auf spezifische Kundensegmente, gezieltes Produktportfolio und hohe Verarbeitungskonsistenz mit notwendiger Qualität. Die Einfärbung durch den Kunden selbst (Self Coloring Concept Colorflexx), starke Kundenbeziehungen und Unterstützung des Vertriebs. In Antwerpen wurde mit einer dreistelligen Millionen-Investition die größte, weltweit führende und kosteneffiziente Produktion errichtet (single strain ABS Plant). Die Vorgabe des verantwortlichen Managers war die 3x10-Regel: 10 Produkte, 10 Tage Inventory, 10 Zahlungskonditionen sowie 100 Prozent E-Commerce.

Wichtig ist die klare und neue Value Proposition. Zentral ist dabei die hoch konstante Qualität des ABS in den Verarbeitungsprozessen des Kunden. Dabei beschränkte sich BASF auf das Segment der „injection moulding applications“.

Für die Selbstfärbung auf den Maschinen der Kunden entwickelte BASF gemeinsam mit den führenden MasterbatchProduzenten (zum Beispiel Clariant) und den besten Herstellern von Dosierungsausrüstungen eine kundenorientierte und verständliche Lösung (inkl. klaren Servicestandards), behielt 
aber gegenüber Kunden die volle Verantwortlichkeit. Damit folgt das Unternehmen dem Prinzip der Variantenbewältigung, dass die Differenzierung möglichst nahe beim Kunden oder sogar beim Kunden selbst erfolgen soll. Sowohl Kunden als auch BASF werden damit gleichzeitig flexibler. Kunden investierten 5.000 bis10.000 Euro pro Maschine. Nur in Ausnahmefällen mit Großkunden engagierte sich BASF für diese Investitionen und kompensierte über langfristige Verträge mit den Kunden.

Change: Die neue Strategie zeigt bereits wichtige Lösungen, die entwickelt wurden. Wichtige weitere Hinweise zum Prozess der Veränderung sind:

- Die Kommunikation nach innen spielte eine große Rolle; einerseits waren die Einsicht und Bereitschaft durch die bestehenden Misserfolge in der Ausgangslage der Führungskräfte und Mitarbeiter groß, anderseits bestand immer die $\mathrm{Ge}$ fahr, wieder in die alte Sichtweise zurückzufallen, der Change-Prozess der Mitarbeiter war anspruchsvoll, gleichzeitig nahm aber auch BASF klar die soziale Verantwortung wahr.

- Die Kommunikation der neuen Lösungen wurde intensiv gewichtet. Dokumentationen, Internet und so weiter erklärten das neue Konzept, die Vorteile für Kunden und dass dieses Konzept bei erfolgreichen Kunden funktioniert. Im Internet konnte sich der Kunde einfach seine Kostenvorteile mit einer Umstellung auf „self coloring" berechnen.

- Der Außendienst wurde geschult und mit Hilfen für die neue Argumentation unterstützt, um die Kunden richtig für eine Umstellung beraten zu können; eine technische „Task Force" stand für besondere Herausforderungen bei Kunden zur Verfügung.

- Die Dauer der Entscheidungsprozesse des Kunden wurde unterschätzt und verzögerte die Umstellung um ein bis zwei Jahre. Auch Kunden, die umstellen wollten, beanspruchten für die neue Arbeitsteilung mit BASF und reale Umstellung recht viel Zeit. Die Änderung für den Kunden ist auch relativ groß.

- Im ersten Jahr führte BASF parallele Angebote, dann wurden die Sortimente endgültig bereinigt.

- Die Auftragsabwicklung wurde konsequent automatisiert und rationalisiert (deshalb die Vorgabe 100 Prozent E-Commerce). Dies entlastete den Verkauf und öffnete seine Spielräume für wichtige Themen und Beziehungen bei Kunden.

Ergebnisse: Bereits 2005 betrieb BASF nur noch zehn (statt den früheren 1.500 Produkten). BASF gewann 30 Prozent der
Kunden bis 2007 neu, die Verluste bewegten sich in der Größenordnung der normalen Fluktuation. Zwischen 2004 und 2007 wurde das Volumen von 100 (indexiert) auf 270 gesteigert. Die Rentabilität wurde erhöht und der Bereich schreibt heute schwarze Zahlen. Die Vorgaben zu Inventory und Zahlungskonditionen wurden erreicht. Die Wirkung in der Branche war enorm. 2001 bis 2007 traten vier von neun Europäern aus diesem Teilmarkt aus, obschon ursprünglich besonders die asiatischen Wettbewerber anvisiert waren. Bei den verbleibenden Anbietern schlugen zwei ebenfalls eine Commodity-Strategie ein, zwei Anbieter setzten auf eine noch intensivere Nischenpolitik mit Mengenverlusten. Der verbleibende Anbieter verfolgt eine gemischte Strategie.

Das konsequente Geschäftsmodell ließe sich durch eine organisatorische Trennung noch konsequenter umsetzen. Die Commodity-Strategie (Glück 2007) soll auch auf weitere Geschäftsbereiche von BASF übertragen werden. Allerdings wird in manchen Sektoren auch weiter das Nischenmodell mit Leistungspaketen für Kunden besser passen.

\section{Differenzierung: Bei einer Differenzierung integrieren} bestehende Anbieter das Volumengeschäft in der bestehenden Organisation. Risiken sind beispielsweise Komplexität, Kompromisse, Gefährdung des Mehrwertgeschäfts. Chancen liegen in einem stufenweisen Vorgehen und möglichen Synergien. Diese Option scheint besonders spannend. Interessant ist auch, dass sich zwischen Volumengeschäft und Kleinkundenmanagement viele Analogien nutzen lassen. Beispielsweise differenzieren Anbieter wie die Swisscom oder die Schweizerische Post ihr Marketing und Vertrieb für kleine und mittlere Unternehmen (Belz et al. 2014).

Manche Unternehmen unterscheiden auch zwischen ihrem eigenen Angebot und sie sind parallel als Original Equipment Manufacturer oder Anbieter von Private Labels aktiv. Auch wenn die Umsatzanteile dieses Geschäfts oft erheblich sind, kommunizieren viele Firmen kaum dazu. Sie flankieren gleichsam das Geschäft unter den eigenen Marken im „Dunkeln“.

4. Realisierung eines Preisbands von 40 Prozent: Um gegenüber preisbewussten Einkäufen der Kunden und preisaggressiven Wettbewerbern zu agieren, kann sich ein Unternehmen auch darauf konzentrieren, den eigenen Preisspielraum auf rund 40 Prozent zu steigern und damit die unterschiedliche Preisbereitschaft der Kunden zu treffen. Dabei sind diese 40 Prozent als mögliche Bandbreite genannt. Sie kann sich je nach Unternehmen und Markt unterscheiden, es geht aber um eine maßgebliche Erweiterung. Vorteil dieses 
Ansatzes ist es, dass die Anbieter ihre Qualität für Kunden vielfältiger interpretieren, aber den angestrebten Höchstleistungen und damit ihrer Strategie treu bleiben können.

Aus allen Ansätzen und Beispielen lässt sich lernen. Auch kann sich im Zeitablauf verschieben, was im Mehrwert- und Volumengeschäft getrennt oder verbunden wird. In der Flugindustrie entstanden früher verschiedene, spezialisierte Billig Carrier. Vieldiskutierte Beispiele waren Southwest, Ryan Air oder EasyJet, die zuerst als Holzklasse-Fluggesellschaften tituliert wurden und den Markt kräftig schüttelten. Die deutsche Lufthansa übernahm aber inzwischen den Konkurrenten Germanwings. Ohnehin lernten die klassischen Anbieter selbstverständlich, mit dem Preisdruck umzugehen. Differenzierte Services, Kostenmanagement und Auslastungsprognosen spielen in ihren Preissystemen eine wichtige Rolle. In der Folge werden die Optionen Differenzierung und Preisband vertieft.

\section{Differenzierung - Lancierung einer parallelen "No-Frills-Linie"}

Es gibt ohne Zweifel schlanke und erfolgreiche Billig- oder Volumenanbieter. Sie verfolgen ein konsequentes Geschäfts- modell, wie es auch andere Modelle gibt. Besonders interessant bleibt aber die Frage: Wie kann ein Unternehmen parallel zum bestehenden Mehrwert-Angebot eine Volumen- oder No-Frills-Linie einführen?

Im praktischen Fall für ein Unternehmen der Medizinaltechnik diskutierten wir die strategischen Variablen in Tabelle 1. Offensiv wäre es, sämtliche Bereiche des Volumengeschäfts vom bestehenden Angebot zu trennen (rechte Spalte). Bei jedem Hebel lässt sich aber prüfen, wie stark das Geschäft mit Billigangeboten vom Bestehenden getrennt oder verbunden werden soll. Typisch etwa die Frage, ob der gleiche Außendienst in der Lage wäre, bei seinen Kunden die Premium- und Volumenangebote zu fördern. Auch mit dem Preisrange, der Markenführung und Produktbezeichnungen, den Produkten und Services, der Selektion der Kunden und Absatzmärkte, den Kanälen, der Struktur und dem Reporting lässt sich differenziert umgehen. Auf diesem Spielfeld sind viele konkrete Kombinationen für Lösungen möglich.

Gegenwärtig folgert das Unternehmen, dass ein BilligpreisAnsatz nur selektiv für besondere Produkte und für ausgewählte Länder verfolgt werden soll. Zwar ist das Vorgehen we-

Tab. 1 Hebel für ein integriertes oder getrenntes Volumengeschäft

\begin{tabular}{|c|c|c|c|}
\hline Strategy & Slow \& passive & Selective & Offensive \\
\hline Price range & $-20 \%$ overall & $-30 \%$ overall & $-50 \%$ lean line \\
\hline Branding & One brand & Mixed portfolio & Lean line brand \\
\hline Product & $\begin{array}{l}\text { One assesment with competitive } \\
\text { products }\end{array}$ & Selective lean line & Broad lean line \\
\hline Service & Incorporated services & $\begin{array}{l}\text { Selective services after the } \\
\text { willingness of the customer to pay }\end{array}$ & Lean line with lean services \\
\hline Customers & Price oriented key accounts & Price oriented customers & $\begin{array}{l}\text { Lean interaction modell for all } \\
\text { customers }\end{array}$ \\
\hline Sales & Sales for all prdoucts & $\begin{array}{l}\text { Different sales organisations with } \\
\text { intense cross selling }\end{array}$ & $\begin{array}{l}\text { Separated and competitive sales } \\
\text { organisations }\end{array}$ \\
\hline Channel & Sales and intende sales support & Multi-Channel & Internet sales modell \\
\hline Countries & $\begin{array}{l}\text { Mono approach in selectid } \\
\text { countries (e. g. Turkey) }\end{array}$ & Dual strategy in selected countries & World wide dual strategy \\
\hline Structure & Integrated organisation & Separation of selected functions & $\begin{array}{l}\text { Separated departments for high } \\
\text { and low approach }\end{array}$ \\
\hline Reporting & Integrated goals and controlling & $\begin{array}{l}\text { Clear differentiated goals and } \\
\text { controlling }\end{array}$ & Separated goals and controlling \\
\hline
\end{tabular}


niger risikoreich, gleichzeitig aber auch weniger grundsätzlich und mutig. Immerhin konkurrenziert das Unternehmen sein aktuelles Geschäft nicht rascher als nötig: Befürchtete Effekte der Eigenkonkurrenzierung werden minimiert.

Besonders ist auch das Beispiel von Kärcher, speziell bekannt für seine Hochdruckreiniger. Vor Jahren baute das Unternehmen neben dem angestammten B2B-Geschäft ein erfolgreiches B2C-Geschäft (beispielsweise mit Baumärkten) auf.

Eine Erfolgsgarantie gibt es aber nicht. Die Lienhard Office Group lancierte mit der Marke Nivo eine günstige Linie für Büroeinrichtungen (Meili 2015). Diese Marke wurde selektiv an ausgewählten Standorten vertrieben. Der italienische Lieferant erwies sich bald nicht mehr als genügend günstig, um einen relevanten Preisunterschied oder die erforderliche Marge zu stützen. Auch waren nur einzelne Produkte der Linie erfolgreich und der Verkauf lernte nicht genügend rasch, mit dualen Angeboten umzugehen. Umso mehr, als die Bezeichnung „dual“ die Situation stark vereinfacht, denn das Premiumgeschäft schließt bereits viele Marken und Varianten mit ein. Zusammenfassend war die Initiative weniger erfolgreich als erwartet und wird auf eine neue Basis mit neuen Lieferanten gestellt.

Im Beispiel ist auch der Verkauf angesprochen. Kann der gleiche Außendienst No-Frills- und High-End-Angebote verkaufen? Was sind die Voraussetzungen für den Erfolg? Die Antwort lässt sich nur im spezifischen Fall bestimmen. Wie die Bezeichnung Volumengeschäft aber bereits klarmacht: Es geht darum, neben dem selektiven hochwertigen Angebot und einer intensiven Kundenbetreuung viel mehr Kunden zu bearbeiten, und in der Regel braucht es auch einen schlanken, aktiven bis aggressiven Verkaufsansatz. Das wird oft auch Flächenvertrieb genannt.

Voraussetzung für den Erfolg bleibt, dass ein Unternehmen in der Wertschöpfung für den Kunden wirksam einsparen und die Preise senken kann, ohne den Erfolg zu schmälern. Dabei spielen selbstverständlich nicht nur die Margen eine Rolle, sondern es gilt, die Mengen zu steigern und Vorteile der Standardisierung zu nutzen.

\section{Realisierung eines Preisbands von 40 Prozent}

Manche Unternehmen sind es bereits gewohnt, ihr Angebot je nach Kunden zu dimensionieren. Typisch sind Informatikund Telekom-Unternehmen, Berater, Trainer und Dienstleister im Marketing, falls sie sich nicht innerhalb von Ausschreibungen bewegen. Auch können sie beispielsweise ihre Vorschläge eher als langfristiges Mandat oder als ein Projekt einbringen. In weiteren Unternehmen ist es möglich, mit Lö-

\section{Handlungsempfehlungen}

1. Überprüfen Sie kritisch, wie Ihr Unternehmen grundsätzlich auf Billigkonkurrenz reagieren kann.

2. Legen Sie sorgfältig fest, wie Ihr Unternehmen die Geschäfte für Mehrwert und Volumen oder „NoFrills"verbindet oder trennt.

3. Erfassen Sie die Qualitätsbedürfnisse und Erwartungen der Kunden und senken Sie gezielt die Kosten durch Varianten von Produkten, Services, Verfügbarkeit, Distribution, Zusammenarbeit, Margen und Umlagen sowie Mengeneffekte. Erweitern Sie Ihren erfolgreichen Preisspielraum auf 40 Prozent.

sungen aus verschiedenen Sparten einen Preisspielraum zu öffnen. Sie stützen sich etwa auf unterschiedliche Maschinentypen oder Technologien, um die Anforderungen des Kunden zu treffen.

Es gilt, Preisspielräume zu öffnen. Hochleistung für Kunden ist differenziert und braucht nicht unbedingt getrennte Ansätze für „Low- und High End“. Woher holt aber ein Unternehmen allgemein einen Preisspielraum von beispielsweise 40 Prozent? Je nach Wettbewerbssituation und Kundenerwartungen lässt sich das Preisband auch anders bestimmen. Das Vorgehen ist jedem Programm zur Kostensenkung ähnlich, allerdings geht es nicht um eine gesamte Senkung, sondern um den differenzierten Umgang je nach Kunde. Nützlich ist auch das Vorgehen nach Kim und Mauborne (2005) in ihrem Buch „Blue Ocean Strategy“, sie plädieren dafür, die aktuell und zukünftig wichtigen Nutzenkomponenten für Kunden zu erfassen und gezielt Werte zu eliminieren, zu reduzieren, zu halten oder zu kreieren. Was gestrichen und vermindert werden kann, interessiert uns hier besonders, weil viele Anbieter ihre Leistungen laufend erweitern.

In jeder Stufe der Wertschöpfung des Unternehmens für Kunden lässt sich einsparen (siehe Abbildung 3). Wichtig ist es dabei, die Wertschöpfung auf Kundensegmente oder noch besser typische Kundensituationen auszurichten und nicht nur von Leistungen und Angeboten auszugehen. Ergiebig ist es auch, für jede Stufe bereits die erreichbare Nettoeinsparung einzusetzen. Investitionen, Entwicklungs- oder Komplexitätskosten lassen sich damit bereits berücksichtigen. Folgende Hebel können eine Rolle spielen und auch hier sind die Möglichkeiten der Einsparung je nach Unternehmen verschieden: 
1. Schlanke Produkte: Der Anteil der Kosten für Produkte ist für Industrieanbieter oft so groß, dass nur eine Einsparung in diesem Bereich auf ein Preisband von 40 Prozent hoffen lässt. Plötner (2012, S. 53 ff.) konzentriert sich deshalb auf schlankere Produkte, etwa durch den Einsatz alter Technologien, die neu oder für besondere Länder produziert werden; durch den aktiven Handel mit Gebrauchtprodukten; umfassende Retrofit-Angebote für bestehende Anlagen der Kunden; De-featured Premiums, die sich auf die Kernleistung konzentrieren und sophistizierte Features streichen; Frugal Engineering, gestützt auf den Kernnutzen der anvisierten Kunden in Massenmärkten. Diese Entwicklung von neuen Produkten für neue Kunden scheint besonders erfolgsträchtig, wenn auch aufwendig. Nach Plötner ist es eine Voraussetzung, die neuen Leistungen nahe bei den anvivisierten Kunden und in ihren Märkten zu entwickeln: „No-Frills-Technologien in Form frugaler Innovationen zwingt Premium-Hersteller dazu, ihre organisationalen Strukturen zu dezentralisieren beziehungsweise zu globalisieren“ (Plötner 2012, S. 82).

„Die Güte der Materialien, Produktlebensdauer, Fehlertoleranzen, Garantie, Sicherheitsstandards, Flexibilität der Einsatzmöglichkeiten und das äußere Erscheinungsbild sind nur einige Variablen, nach denen Kunden die Qualität eines technischen Produktes bewerten“ (Plötner 2012, S. 65). Hier bestehen die Möglichkeiten zur Kostensenkung, wenn Kundengruppen ihre angestrebte Qualität in ihrem Einsatz verschieden beurteilen. Angestrebt sind Leistungen, die genau treffen. Klassische Diskussionen in Unternehmen kreisen auch um Basisausstattungen, Standardisierung, modulare Produkte, Vereinfachung und Rationalisierung. Interessant ist auch das Vorgehen beim Kunden, werden schlanke Lösungen schrittweise mit ihm erweitert oder werden Features bei umfassende Produkten abgewählt? Diese Vorgehensweisen werden in Konfiguratoren, beispielsweise bei Autos, vielfach erprobt.

Abb. 3 Preisband von 40 Prozent durch Einsparungen

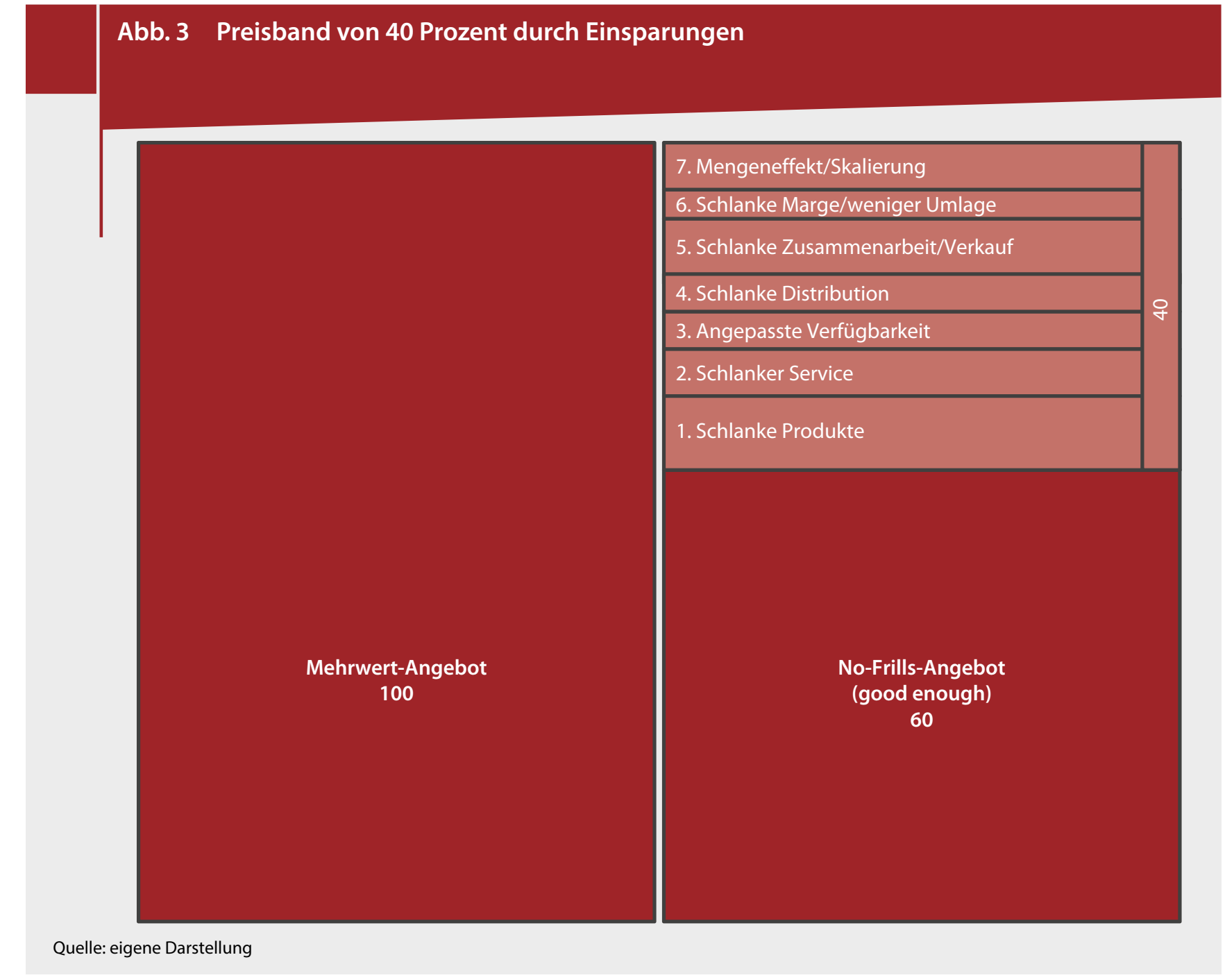


Target Pricing soll die neuen Entwicklungen konsequent auf den angestrebten Preis bei guter Marge des Unternehmens richten. Auch ist es besonders in Unternehmensgruppen oft möglich, die Kundenlösungen aus verschiedenen Sparten mit verschiedenen Technologien und Maschinen vorzuschlagen und dabei große Kosten- sowie Preisdifferenzen zu erzielen.

Schließlich stellen sich auch die grundsätzlichen Fragen zu den richtigen Produktionsstandorten, die ganz verschiedene Ressourcen und Kostenfaktoren haben können. So lassen sich in einem Werk in Indien maßgebliche Kostenvorteile realisieren. Die Risiken sind aber erheblich, die Logistik ist zu lösen und auch die Akzeptanz von „Made in India“ der Kunden spielt eine Rolle.

2. Schlanker Service: Auch der Service lässt sich auf das Notwendige ausrichten und abspecken. Typisch ist die Arbeit mit Service Levels für Kunden. Auch lassen sich die Serviceoptionen generell für Kunden zur Wahl stellen und getrennt verrechnen.

Grundsätzlicher ist die Herausforderung, dass schlanke Produkte oft auf Märkte stoßen, die Serviceleistungen und damit verbundene Abhängigkeiten von Lieferanten ablehnen. Im Extremfall müssen Anbieter bereits mit dem Erstverkauf von Maschinen oder Lösungen und ohne Service erfolgreich sein. Das widerspricht den Premium-Geschäftsmodellen, weil sie die Erträge dort besonders nach dem Kauf der Kunden erwirtschaften (Plötner 2012, S. 47 ff.), denn der EBIT ist in vielen Unternehmen im Service weit höher als im Produktgeschäft.

3. Angepasste Verfügbarkeit: Anbieter mit hohen Fixkosten und spezifischen Kapazitäten sind stark von ihrer Auslastung abhängig. Gelingt es, diese Auslastung zu steuern, so sind damit große Einsparungen möglich. Die Voraussetzung ist es aber, dass sich mit den Kunden über sinnvolle Verfügbarkeit und Lieferfristen diskutieren lässt. Die klare Lösung bestünde hier in einem Yield Management mit auslastungsabhängigen Preisen, wie sie die Flugbranche, Hotels und viele mehr schon lange praktizieren. Im Handel mit Materialien ist das Spotgeschäft mit günstigen Konditionen verbreitet. Ist der Kunde bereit, sich anzupassen, erreicht er maßgebliche Preisvorteile. So unterscheidet beispielsweise SBB Cargo (das Logistikunternehmen der Schweizerischen Bundesbahnen) zwischen zeitkritischer und zeitunkritischer Logistik bei Kunden und erwartet daraus wichtige Optimierungschancen.

4. Schlanke Distribution: Der persönliche Verkauf ist national und international sehr aufwendig. Wenn es mit dem Internet gelingt, die Beratung und Betreuung zu standardisie- ren und gleichzeitig für Kunden zu verbessern, so ist es möglich, weit effizienter anzubieten. Die Verbesserung schließt dabei nicht nur die inhaltliche Beratung ein, sondern ebenso die Sprache des Kunden, die zeitliche und örtliche Verfügbarkeit, den gleich hohen Standard, die Zuverlässigkeit sowie Geschwindigkeit. Funktionalitäten zum Erfahrungsaustausch zwischen Kunden, zu Anwendungen mit kurzen Filmen, zu präventiver Wartung und Teleservice und so weiter erweitern die Möglichkeiten.

Typisch auch der Hinweis zum E-Commerce von Karl Spachmann (Leiter internationaler Vertrieb von Geberit im Gespräch am 12.06.2015): Seltene Käufe sind bei Kunden immer öfter durch Internet-Recherchen vorbereitet. Auch wenn der Internetanteil zukünftig bei sieben Prozent liegen wird, haben kleinere Internethändler eine enorme Pricing Power. Internetrecherchen prägen die Preiserwartung. Zudem ist Geberit nicht aktiv im Internet präsent, um bestehende Partner zu schonen. Wenn Geberit zu 100 anbietet, sind präsente Konkurrenzprodukte bei 80 und im Internet bei 56 (-30 Prozent). Alle Partner im Markt werden zunehmend unter Druck gesetzt. Kurz: Neue Lösungen sind gefragt.

5. Schlanke Zusammenarbeit/schlanker Verkauf: Nicht jeder Kunde braucht eine extensive Zusammenarbeit, und einzelne Kunden beanspruchen einmal eine umfassende Unterstützung und bei einem nächsten Auftrag eine sehr schlanke Zusammenarbeit. Unternehmen mit einer Monoleistung (also zum Beispiel mit dem erwähnten, aufwendigen Mehrwertangebot) werden vom Kunden nur für spezifische Einkaufssituationen beigezogen und in weiteren ausgeschlossen. Um zu wachsen und Erträge zu steigern, versuchen deshalb Unternehmen mehr und mehr, etwa drei bis sechs verschiedene Zusammenarbeitsvarianten von extensiver bis schlanker Zusammenarbeit mit Kunden zu beherrschen (Belz 2015). Angestrebt ist es dabei, die Zahlungsbereitschaft des Kunden zu treffen mit dabei ähnlich attraktiven Margen im schlanken Volumengeschäft wie im extensiven Geschäft.

Damit definieren sie ein differenziertes Zusammenarbeitsportfolio, aber sie gehen keinesfalls für jeden Kunden individuell vor. Relevant ist das angestrebte Mengengerüst für die Interaktionsmodelle. In jedem Modell spielen „Economies of Scale" eine Rolle. Es ist sinnvoll, die Zahl der Zusammenarbeitsstufen gering zu halten, denn Verkäufer und Kunden sind rasch überfordert. Die Interaktionsmodelle müssen aber gleichzeitig die vielfältige Zusammenarbeit mit Kunden einigermaßen konkret erfassen, das führt zu einer stärkeren Differenzierung. 
So unterscheidet beispielsweise BASF weltweit sechs Interaktionsmodelle vom Trader/Transactional Supplier (Käufer brauchen standardisierte Produkte in transparenten Märkten) bis zum Value Chain Integrator (Kunden sind bereit, ihre eigene Value Chain zu öffnen, und erkennen eine substanzielle Kosten- und Risikoreduktion in einer umfassenden Delegation ihrer Aufgaben und Zusammenarbeit) (Kramer 2012). Zwar treffen nicht alle sechs Interaktionsmodelle auf jede Sparte und jedes Land zu, die Komplexität ist jedoch für Unternehmen und Vertrieb groß. Überschaubarer ist das bereits langfristig gepflegte Modell von Bossard (Händler für Befestigungstechnik). Die drei Stufen zeigt Abbildung 4 (Grob 2014).

Damit ist das Unternehmen als schlanker Lieferant für standardisierte Schrauben (Produkte) ebenso wettbewerbsfähig wie als Partner für innovative Befestigungen (Engineering) oder die umfassende Kleinteilelogistik der Kunden (Logistik).

Plötner (2012, S. 82) fordert, die Premium- und No-FrillsAngebote in getrennten Vertriebsteams zu vermarkten. Die No-Frills-Technology-Vertriebler müssen verkaufs- und nicht beratungsorientiert sein. Dabei kann es sich um unternehmenseigene Teams handeln, die variabel entlohnt und deren Arbeit durch Online-Vermarktung ergänzt wird, oder um Handelspartner (Plötner 2012, S. 71). Diese Frage gilt es jedoch, spezifisch zu lösen, und weitere Forschung dazu ist wichtig. Beispielsweise ist in größeren Märkten eine weitere Spezialisierung möglich. In kleinen Märkten werden Generalisten im Verkauf eingesetzt.

Mit flankierender Marktbearbeitung lässt sich der Verkauf entlasten und das Gesamtsystem optimieren. Dabei ist es nö- tig, so weit wie möglich zu standardisieren, um erst dann mit einer professionellen Individualisierung für Kunden aufzusetzen.

6. Schlanke Marge/weniger Umlage: Grundsätzlich will ein Anbieter seine Erträge über alle Bereiche optimieren. Trotzdem kann es sinnvoll sein (besonders im Zusammenhang mit 7.), im Volumengeschäft die Marge zu mindern.

Besonders bei komplexen Angeboten ist die Kosten-PlusMethode fest in den Köpfen der Verantwortlichen verankert. Daran ändert auch wenig, wenn mit einem Margenaufschlag gearbeitet wird. Nicht selten werden Kostenvorteile (beispielsweise im Einkauf) direkt in Preisvorteile für Kunden umgerechnet. Die Alternative besteht darin, die Preise nutzenorientiert und psychologisch zu gestalten. Nur ist dieses Feld weniger klar fassbar als reine Kostenbetrachtungen. Besonders in den komplexen Offerten sind Preispsychologie und Nutzen schwieriger einzubeziehen, aber nicht weniger wichtig. Flankiert wird der erwähnte Aspekt durch die interne Preispsychologie. Häufig stellt sich die Frage, wie weit die Verkaufsverantwortlichen die Kosten kennen müssen. Manche Unternehmen folgern, dass die Kostentransparenz der Mitarbeitenden systematisch zu tieferen Preisen führt. Kurz: Sowohl im Mehrwert- als auch im Volumenbereich braucht es eine professionelle Preisgestaltung. Daraus ergeben sich auch die angestrebten Unterschiede.

Zudem ist die Umlage der Gemeinkosten zu überdenken. Die Umlage hoher Fixkosten sollte ein neues Volumengeschäft nicht verhindern. Manche Infrastrukturen sind auch eindeutig nur für das Mehrwertgeschäft, aber nicht für das

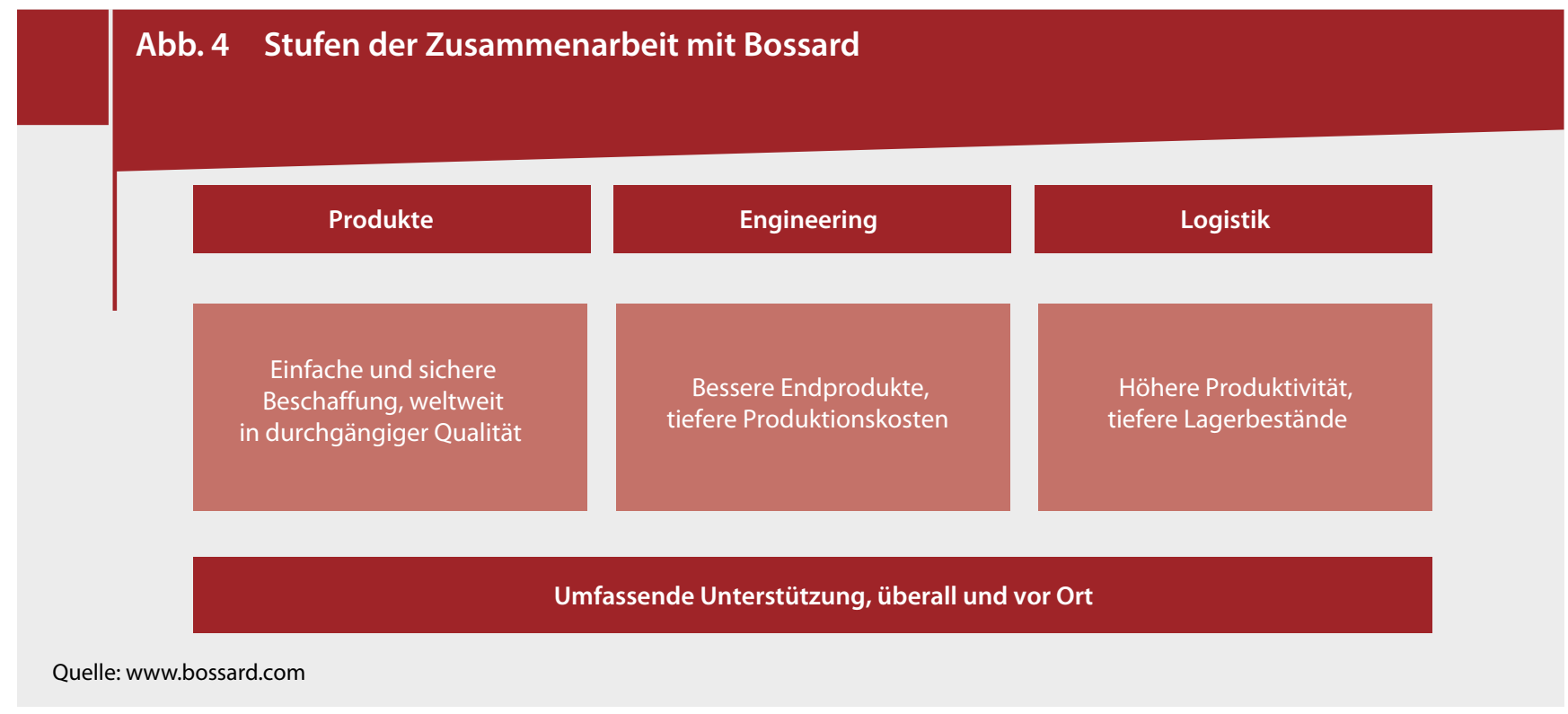


Massengeschäft nötig. Andererseits diskutieren vorwiegend erfolglose Manager ständig über die internen Umlagen und „wer zahlt was“.

7. Mengeneffekt/Skalierung: Wie die Begriffe Volumen und Standard andeuten, geht es um Menge und Skalierung. Sie beeinflussen die Kosten maßgeblich, sind aber auch schwierig zu prognostizieren.

Wie weit eine Preisdifferenzierung 100/60 durchgezogen wird, lässt sich verschieden gestalten. Mehrere Zwischenstufen von 60 bis 100 sind möglich.

Ein Ansatz besteht darin, den wichtigen, potenziellen Kunden zwei alternative Lösungen innerhalb des eigenen Unternehmens vorzuschlagen, vorzugsweise mit unterschiedlichen Preisen auf dem Niveau von 60 und 100. Damit kann der Kunde bereits wählen und wird weniger Wettbewerber einbeziehen. Der Anbieter sucht aktiv danach, mögliche Preisspielräume mit verschiedenen Lösungen zu verbreitern. Er stützt sich nicht auf Vermutungen, welches Preis- und Leistungsangebot für Kunden richtig liegt. Natürlich ist dabei zu entscheiden, bei welchen Kunden dieses Vorgehen richtig ist. Den Kunden wählen zu lassen, ist nicht unkritisch. Erstens ist er rasch überfordert und zweitens kann der Anbieter mit der Wahl verlieren.

Manche Anbieter werden die Varianten auch nur intern grob evaluieren und versuchen zuerst, mit einem Angebot zum Abschluss zu kommen. Sie behalten die zweite Variante in der Hinterhand. Allenfalls lässt sich die Positionierung auch grob mit den Kunden abklären. Welches Budget haben sie sich vorgestellt? Diese Frage ist möglich, aber auch kritisch. Die Hinweise des Kunden bleiben als Anker bestehen und sind, falls nötig, umso schwerer zu korrigieren.

\section{Fazit}

Eine Premiumposition lässt sich in neuen Ländern oder mit zusätzlichen Leistungen ausbauen. Neue Länder im angestammten Bereich zu erschließen, hat andere Risiken und Chancen als eine parallele Volumenstrategie.

Ob sich ein Mehrwert-Anbieter im Massenmarkt engagieren soll, ist eine strategische Entscheidung. Ein neues Geschäftsmodell steht zur Diskussion. Zwar ist es möglich, neue und getrennte Unternehmen für den Massenmarkt zu übernehmen oder aufzubauen, aber integrierte Ansätze und größere Preisspielräume liegen näher.

Die Situationen in den Märkten und den Unternehmen unterscheiden sich maßgeblich. Ein Standardvorgehen für das Standardgeschäft gibt es nicht. Es geht um spezifische und stimmige Gesamtkonzepte und vor allem die Realisierung.
Und: Unternehmen lernen aus Initiativen im Volumengeschäft auch für ihre angestammten Mehrwertangebote.

\section{Literatur}

Baumbach, M. (2004): After Sales Management im Maschinen und Anlagenbau, Universität St. Gallen: Dissertation.

Belz, Ch. (2015): Neue Formen der Zusammenarbeit mit Kunden in der Industrie - Wann lohnt sich eine intensive Zusammenarbeit mit Kunden?, in: Hoffmann, Ch. P. et al. (Hrsg.) (2015): Business Innovation: Das St. Galler Modell, Wiesbaden (erscheint demnächst).

Belz, Ch. (2009): Marketing gegen den Strom, St. Gallen: Index und Thexis.

Belz, Ch. et al. (2014): Herausforderungen und Stellhebel für die erfolgreiche Betreuung von Kleinkunden, in: Marketing Review St. Gallen 31, 3, S. 14-24.

Christensen, C. et al. (2013): The Innovators Dilemma - Warum etablierte Unternehmen den Wettbewerb um bahnbrechende Innovationen verlieren, München (das amerikanische Original erschien bereits 1997).

De Saint-Exupéry, A. (2000): Wind, Sand und Sterne, 24. Auflage, Düsseldorf (erstmals 1939).

Glück, A. (2007): Präsentationen und Gespräche mit Dr. Andreas Glück (BASF), die Hinweise betreffen nur die Entwicklungs- und Transformationsphase.

Grob, B. (2014): Präsentationen und Gespräche mit Beat Grob (CEO).

Kim, Ch. W./Mauborgne, R. (2005): Blue Ocean Strategy, Boston.

Kramer, M. (2012): Präsentation am Fachdialog für Marketing des Instituts für Marketing der Universität St. Gallen am 05.06.2012.

Levitt T. (1966): Innovative Imitation, in: Harvard Business Review, 44, Sept.-Oct., S. 63-70.

Lüthi, B. (2006): Präsentation von Beat Lüthi (vormals Mitglied der GL Mettler-Toledo) zur Ausgangslage der Strategie (inzwischen hat sich die Konstellation in Unternehmen und Markt verändert (www. ohaus.com und www.mt.com).

Meili, M. (2015): Gespräch mit Markus Meili (CEO Lienhard Office Group) am 12.03.2015 (www.nivo-office.ch und www.lienhardoffice.ch).

Plötner, O. (2012): Counter Strategies im globalen Wettbewerb, Berlin/Heidelberg.

Prahalad, C. K. (2004): The Fortune at the Bottom of the Pyramid.

Vunder, F. K. (2008): Sales Channels for Targeting Base-of-Pyramid Markets in India, St. Gallen: Dissertation. 


\section{Springer Gabler}

Joachim Dorschel Hrsg.

\section{Praxishandbuch Big Data}

Wirtschaft - Recht - Technik

SpringerGabler

\section{Big Data: Chancen und Herausforderungen für Unternehmen}

Joachim Dorschel (Hrsg.)

Praxishandbuch Big Data

2015. 364 S. 40 Abb. Geb.

$€$ (D) $59,99|€(A) 61,67|{ }^{*}$ sFr 75,00

ISBN 978-3-658-07288-9 (Print)

$€ 46,99$ | * sFr 60,00

ISBN 978-3-658-07289-6 (eBook)
- Alles, was Unternehmer über Big Data wissen müssen

- Best Practices geben Einblick in die Anwendung von Big Data in verschiedenen Branchen wie Banken, Telekommunikation, Industrie

- Schwerpunkt Datenschutz und Datensicherheit im Unternehmen

Dieses Praxishandbuch bietet einen Überblick der möglichen Anwendungsfelder und der rechtlichen Rahmenbedingungen von Big Data im Unternehmen. Im ersten Teil wird gezeigt, wie Entscheidungsprozesse mit Daten fundiert werden können und welche Anwendungsmöglichkeiten in verschiedenen Branchen denkbar sind. Der zweite Teil behandelt die rechtlichen Aspekte von Big Data. Die Autoren geben praktische Empfehlungen, wie Big Data-Anwendungen nach geltendem Recht umgesetzt werden können und dabei den technischen und organisatorischen Aufwand so gering wie möglich zu halten. Auch wird erläutert, wie Unternehmen ihre Datenbestände schützen können. Der dritte Teil beschäftigt sich mit den technischen Voraussetzungen von Big Data-Anwendungen.

Jetzt bestellen: springer-gabler.de 